\title{
Structure sociale et ordres juridiques originels dans l'Outaouais supérieur et les régions voisines
}

I - L'organisation sociale des peuples algonquiens du Québec dans la

perspective de la "longue durée"

\section{Estructura social y órdenes legales originales en la región del Outaouais Superior y las regiones vecinas}

I-La organización social de los pueblos algonquinos de Quebec en la

perspectiva de la "larga duración"

\section{Social structure and original legal systems in the Upper Ottawa River and surrounding regions \\ I-The social organization of the Algonquians of Québec from the perspective of the "longue durée"}

\section{Jacques Leroux}

Volume 46, numéro 2-3, 2016

Diversité et altérité d'identité et de territoire

URI : https://id.erudit.org/iderudit/1040439ar

DOI : https://doi.org/10.7202/1040439ar

Aller au sommaire du numéro

Éditeur(s)

Recherches amérindiennes au Québec

ISSN

0318-4137 (imprimé)

1923-5151 (numérique)

Découvrir la revue

\section{Citer cet article}

Leroux, J. (2016). Structure sociale et ordres juridiques originels dans l'Outaouais supérieur et les régions voisines : I - L'organisation sociale des peuples algonquiens du Québec dans la perspective de la « longue durée ". Recherches amérindiennes au Québec, 46(2-3), 105-116.

https://doi.org/10.7202/1040439ar

\section{Résumé de l'article}

Cet article fait un retour sur des thèses classiques en recherche algonquiniste en noyautant la réflexion autour des conceptions de la bande déjà proposées. Il présente d'abord un bref aperçu de recherches antérieures sur une application de la théorie des maîtrises foncières et fruitières où les concepts de bande locale et de bande régionale ont été utilisés et il pose à nouveau le problème de leur définition en reprenant à Edward S. Rogers un questionnement sur des notions afférentes. En prenant pour modèle une classification de niveaux d'interactions sociales proposée par Eleanor Leacock, l'auteur tente d'illustrer la configuration de la maisonnée multifamiliale en se reportant à un récit d'« hivernement » du Père Paul Lejeune, en vue de dégager certains paramètres de conduites sociales caractérisées par l'esprit du don. La mythologie est convoquée pour témoigner de cet esprit chez les Algonquins de l'Outaouais supérieur, pour ensuite servir d'amorce au développement d'une hypothèse sur la bonne distance à établir pour se trouver un conjoint dans le cadre des relations matrimoniales. L'auteur interroge ensuite l'emploi des mots " quartier » et « district » qui auraient désigné des espaces de maîtrises foncières et fruitières étayant les rapports de collaboration entre les membres d'une unité que Leacock appelait « bande d'hiver ».
Tous droits réservés @ Recherches amérindiennes au Québec, 2017

Ce document est protégé par la loi sur le droit d'auteur. L’utilisation des services d'Érudit (y compris la reproduction) est assujettie à sa politique d'utilisation que vous pouvez consulter en ligne.

https://apropos.erudit.org/fr/usagers/politique-dutilisation/ 


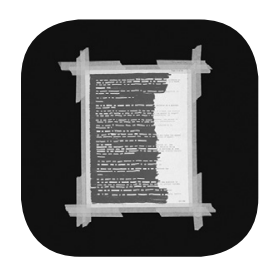

Structure sociale et ordres juridiques originels

dans l'Outaouais supérieur et les régions voisines

I - L'organisation sociale des peuples algonquiens du

Québec dans la perspective de la « longue durée »

\section{Jacques Leroux}

Département d'anthropologie,

Université de

Montréal
D ES AVANCÉES NOMBREUSES ont été faites depuis une vingtaine d'années dans l'étude des institutions sociales algonquiennes de l'AbitibiTémiscamingue et de la HauteMauricie, nommément celles de MariePierre Bousquet $(2008,2012,2015)$, de Claude Gélinas (1998, 2000, 2003a, 2003b), ainsi que de Leila Inksetter dans sa thèse de doctorat (2015). Ces trois auteurs ont porté leur attention sur la thématique de l'autorité en étudiant les formes de pouvoirs au sein des institutions politiques et religieuses tout en s'efforçant de comprendre, chacun à leur manière, les processus qui les ont modifiées au cours des $\mathrm{XIX}^{\mathrm{e}}$ et $\mathrm{XX} \mathrm{x}^{\mathrm{e}}$ siècles en regard de l'évolution du mode de subsistance et du processus de conversion au christianisme. Je m'en inspirerai pour explorer les principales avenues de recherche qu'ils ont parcourues, mais je le ferai dans un article qui suivra celui-ci et qui pourrait avoir comme titre provisoire « Les assises de l'autorité entre rapports de forces et rapports de sens $»$. Je m'efforcerai d'y dégager certains principes de fonctionnement qui permettraient de mieux comprendre le cours des événements relatifs aux métamorphoses du champ politique et des ordres juridiques originels. Je ne prétends en rien à l'originalité, car je m'inspirerai en cela de plusieurs algonquinistes, mais ce sera pour attirer l'attention sur des processus d'effacement qui, comme je tenterai de le montrer dans des articles ultérieurs, ont eu de graves conséquences sur le plan des relations intersubjectives ${ }^{1}$.

Cela explique que ce texte soit le premier d'une série mis à l'enseigne de la structure sociale et des ordres juridiques. Par la locution «structure sociale » je désigne les dispositifs qui organisent le mode de subsistance (champ de l'économie), les instances vouées à la régulation des rapports susceptibles de produire des tensions à l'intérieur du groupe (champ du politique) et le système de parenté en tant qu'il sert de substrat au déploiement des relations sociales qui sont couplées à la subsistance et à la reproduction du groupe. J'emploierai aussi le vocable de «structure conceptuelle » en le rapportant à des notions de cosmologie et au système rituel en tant que celui-ci organise les rapports mutuels des individus et des collectifs dans leur ouverture au monde vivant (champ du religieux). Quant aux ordres juridiques, je les envisagerai dans un troisième article en fonction de ce que Pierre Legendre appelle le «lien institutionnel ». J'axerai alors ma réflexion sur les transformations survenues au XIX ${ }^{\mathrm{e}}$ siècle dans la terminologie algonquine de la parenté et 
Tableau 1

Maîtrises foncières et maîtrises fruitières chez les peuples algonquiens du Québec

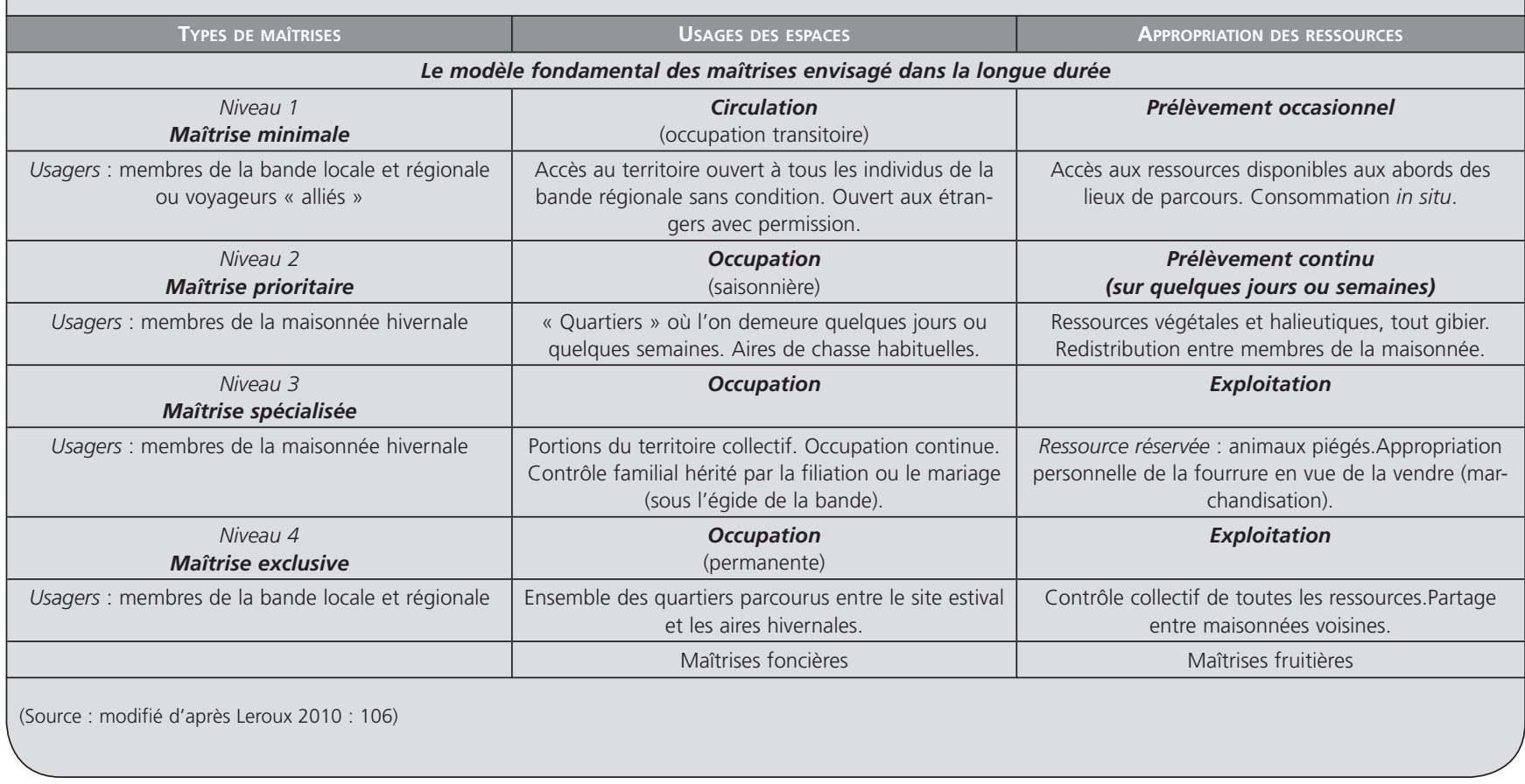

sur les retentissements de ce phénomène sur les conceptions du lien généalogique, en adoptant les perspectives de cet auteur sur ce qu'il appelle la « fonction juridique » :

Fabriquer le lien institutionnel, c'est l'œuvre de la généalogie, qui fait tenir le fil de la vie, rappelle au sujet son assignation dans l'espèce, procure à la société son matériau vivant. L'étude de ce lien aujourd'hui conduit à mettre en rapport le biologique, le social et I'inconscient, à reprendre sur cette base l'observation de la fonction juridique, qui, dans son essence consiste à produire artificiellement le nouage de ces trois indices de l'humain. (Legendre 2004 : 10, les italiques sont de l'auteur)

\section{MAÎTRISES FONCIĖRES, MAÎTRISES FRUITIĖRES ET NIVEAUX D'INTERACTION SOCIALE ENVISAGÉS DANS LA LONGUE DURÉE}

Dans un article que j’ai rédigé au cours des discussions menées avec Étienne Le Roy et Sylvie Vincent (Leroux 2010), je m'étais inspiré des travaux du Laboratoire d'anthropologie juridique de Paris en me reportant aux paramètres d'analyse que notre groupe de travail avait adoptés et sur lesquels nous avons continué à travailler, comme en témoignent leurs textes dans le présent numéro. Ces paramètres reposent sur une distinction clairement établie entre des mâ̂trises foncières, qui se rapportent à des espaces de circulation ou d'occupation, et des maîtrises fruitières, qui se rapportent à des modes de prélèvement et d'exploitation des ressources. Le tableau suivant (tab. 1) présente une vue synoptique des principaux paramètres envisagés en corrélant des acteurs (individuels ou collectifs) qui sont identifiés ici comme
« usagers» ( $1^{\text {re }}$ colonne de gauche) en tant qu'ils auraient des « compétences » (ce qui signifie aussi qu'ils seraient considérés comme des ayants droit) sur des espaces ( $2^{\mathrm{e}}$ colonne) et des modes d'appropriation de la ressource ( $3^{e}$ colonne).

Je me reporterai aux trois auteurs mentionnés en introduction afin d'approfondir l'étude des processus d'interactions sociales qui étayaient les manifestations de l'autorité, mais ce sera dans l'article qui suivra celui-ci. En effet, il m’apparaît nécessaire de revenir au préalable sur quelques textes classiques de l'ethnologie algonquienne en vue de raffiner la description des unités constituantes de la structure sociale, et c'est donc à cela que je m'emploierai dans le présent texte.

\section{DE QUELQUES CONCEPTS FONDAMENTAUX SUR L'ORGANISATION SOCIALE DES PEUPLES ALGONQUIENS}

Bien que le livre de José Mailhot intitulé Au pays des Innus. Les gens de Sheshatshit (1993) n'ait pas encore atteint le quart de siècle, je pense qu'on peut le compter parmi les textes classiques. Je m'en étais partiellement inspiré pour élaborer mon tableau en reprenant ses concepts de « bande locale » et de «bande régionale ». Ce sera le point de départ, et disons donc d'emblée que cette ethnologue les a utilisés en les rapportant à des entités géographiques et démographiques d'échelles différentes:

[Les communautés innues] ont aujourd'hui l'aspect de villages, mais la population qui les habite porte toujours le nom de bandes, hérité de l'époque du nomadisme. Les bandes telles qu'on les connaît 
aujourd'hui sont toutefois un phénomène relativement récent : la plupart d'entre elles sont des groupes hétérogènes, formés autour des postes de traite, qu'on appelle bandes régionales. Antérieurement, I'organisation sociale chez les Innus était basée sur des unités plus petites et mieux articulées qu'on appelle bandes locales. Elles étaient constituées de moins d'une centaine d'individus étroitement reliés entre eux, et chacune occupait un bassin de rivière différent dont elle tirait en général son nom (Rogers 1969; Leacock 1969). Durant la plus grande partie de l'année, elles étaient subdivisées en groupes de chasse [...]. (Mailhot 1993: 53, les italiques sont de moi)

J. Mailhot a aussi montré que ces entités sociales jouent des rôles particuliers selon les régions et les époques en regard de l'échange matrimonial. En effet, avant les années 1950, des subdivisions de groupes occupant des zones bien identifiées à des bassins hydrographiques sont désignées comme bandes locales (ibid. : 55 et 126), mais il faut préciser que certains groupes, comme ceux de la BasseCôte-Nord, «n'ont jamais comporté de subdivisions [et que] le groupe exogame était la bande» (ibid.: 126), le terme «bande» paraissant coïncider ici (en regard de l'échange matrimonial) avec le concept de bande locale. Celle-ci remplissait donc l'emploi d'une unité exogamique, cette fonction se corrélant à plusieurs impératifs, comme celui de donner « la clé d'accès » à de nombreux territoires où les Innus avaient des parents (ibid. : 148). En effet, le cercle de ces parents s'étendait principalement au sein des bandes voisines et celles-ci formaient des groupes d'interactions privilégiées (voir ibid. : 127, la figure 24 qui présente très clairement les aires de distribution et les principaux réseaux de l'échange matrimonial dans l'est de la péninsule du Québec-Labrador avant 1950²). Ces groupes se présentaient donc comme des unités formant des réseaux d'alliances relativement bien circonscrits et certains d'entre eux se recoupaient avec ceux des bandes régionales qui se sont constituées à différentes époques autour des postes de traite. En m'inspirant de la mythologie algonquine, j'avancerai ici l'hypothèse que ce type d'organisation aura aussi existé dans l'Outaouais supérieur et je développerai cette idée sous d'autres considérations dans le deuxième article en rapportant cette unité à ce qui fut appelé ailleurs «macrobande» (Leroux et al. 2004: 16 et 26). Il conviendrait cependant de dégager les autres horizons de ce décor en « revisitant» maintenant les textes d'Eleanor Leacock et d'Edwin S. Rogers qui se sont employés à décrire la composition et le fonctionnement des unités sociales chez les peuples algonquiens dans un colloque consacré aux «band societies ». Ils en ont publié les résultats dans un ouvrage qui porte ces mots en titre, car, en effet, le concept de « bande » y est central (in Damas 1969).

Dans son article, Rogers recense les définitions et les conceptions que divers auteurs en ont proposées en considérant plusieurs sociétés du monde. Il les juge toutes impropres à l'analyse des groupes algonquiens (ce qui m'exemptera d'en discuter - à ce sujet voir Leacock [1969: 3] qui formule un jugement similaire), puis il expose les vues des auteurs ayant spécifiquement travaillé sur les sociétés du Subarctique canadien (Rogers 1969 : 21-24). Là encore, il ne me paraît pas idoine de les discuter toutes et je ne retiendrai que celles qui m'apparaissent les plus importantes. Rogers lui-même était un anthropologue aguerri par une longue expérience de terrain, ayant fait des recherches approfondies chez les Ojibwas de Round Lake en Ontario (1962) et chez les Cris de Mistassini (1963), compétence qui se doublait de connaissances approfondies de l'ethnologie des peuples algonquiens, ces qualités justifiant à mon avis que l'on retienne quelques questions essentielles qu'il a posées pour appréhender adéquatement le problème. Voici comment il les présentait :

\begin{abstract}
Avant qu'il soit possible d'examiner les données relatives à l'organisation et à la structure de la " bande », certains paramètres, qui sont peut-être interreliés et qui permettraient de circonscrire une " bande ", doivent être spécifiés. Ces paramètres sont les suivants: est-ce que des groupes nommés existent? Et, dans l'affirmative, de quoi se compose un tel groupe? Qu'est-ce qui définit les limites du territoire habité ? Existe-t-il à l'intérieur d'un tel groupe quelque homogénéité culturelle qui le distingue d'autres groupes? Quelles sont les relations entre un tel groupe et des groupes similaires? En quoi consiste le procès de fission et de fusion à l'intérieur d'un tel groupe? (Rogers 1969 : 24)
\end{abstract}

Ces questions orienteront la réflexion proposée ici, mais avant de les reprendre en regard des groupes de l'Outaouais supérieur (prioritairement) et des régions voisines (secondairement), je prendrai comme modèle de composition sociale celui qu'a tenté de reconstituer Eleanor Leacock dans son article sur la bande des « Montagnais-Naskapi » (1969: 1-20). Ce procédé s'explique d'abord parce que cette ethnologue s'est efforcée de construire le modèle qui aurait prévalu avant et durant les premières décennies qui mirent les Innus en présence des Européens, et il pourrait donc présenter des recoupements intéressants avec le modèle qui prévalait chez les Algonquins et les Attikameks à l'époque où se présentèrent les missionnaires oblats. Bien que ces rencontres s'y produisirent avec deux siècles de retard et que les postes de traite y connurent des implantations parfois plus tardives, je postule que des homologies de structures très anciennes auront perduré à l'intérieur de tous ces groupes jusqu'au début de la période considérée. Ce détour par les Innus s'impose au demeurant parce que la culture et l'organisation sociale de ce peuple ont été beaucoup mieux documentées et plus profondément étudiées que celles des Algonquins et des Attikameks : par les missionnaires jésuites, d'abord, et par les ethnologues, ensuite. Ce détour s'effectuera aussi en passant par la population de Mistassini, proche parente des Innus, où Rogers (1963), comme on l'a vu, et Tanner (1971 et 1979), ont mené d'importantes études.

Je développerai mon commentaire en illustrant la composition et le fonctionnement des maisonnées hivernales 
dans leur rapport aux unités du même ordre de grandeur en suivant le texte du jésuite Paul Le Jeune pour tenter ensuite de retrouver les unités qui auraient rempli des fonctions équivalentes chez les Cris, les Algonquins et les Attikameks. En postulant que des schèmes fondamentaux ont perduré dans la longue durée (c'est-à-dire, en l'occurrence, depuis l'ère des premiers contacts jusqu'à aujourd'hui), j'amorcerai la description des collectifs à travers lesquels s'organisait le rapport social en vue de situer les schèmes de communication qui régissaient l'exercice de l'autorité et l'éthique du sujet, étude qui sera plus amplement développée dans le deuxième article.

Ainsi, Leacock (1969) identifiait quatre grands types de « groupements», sa nomenclature correspondant à un ordre de grandeur croissant :

1. The multi-family lodge group (dimension: 10 à 20 personnes). Le syntagme multi-family peut se traduire par le mot multifamilial et je pense que l'on traduirait adéquatement le segment lodge group par le terme maisonnée puisque ce mot désigne à la fois une demeure (un logis) et ses habitants, soit «l'ensemble de ceux qui habitent la même maison» (Petit Robert 1972: 1026). Je traduirai donc l'énoncé entier par la locution maisonnée multifamiliale.

2. The aggregate of several lodge groups, or the winter band (dimension: de 35 à 75 personnes). Comme nous le verrons, ce groupement se contracte et se dilate au gré des bonnes ou des mauvaises chasses, aussi bien que de façon régulière et planifiée à certains moments de l'hiver. On pourrait donc l'envisager comme un collectif à géométrie variable, plutôt que comme une unité parfaitement distinctive. Le plus simple sera de l'appeler provisoirement «bande d'hiver», mais nous verrons que nous pourrions rapporter ce collectif à des groupements qui se manifestent aussi durant les trois autres saisons.

3. The named group commonly called the band (dimension: de 150 à 300 personnes). Cette dénomination peut se traduire mot pour mot : le groupe nommé communément appelé la bande. Comme nous le verrons, le "groupe nommé » peut désigner aussi bien la bande locale que la bande régionale (d'apparition plus récente), voire une unité correspondant à ce que l'on appelait la «nation » dans certains écrits des XVII et XVIII ${ }^{\mathrm{e}}$ siècles.

4. Gathering of people from several bands (dimension: près de 1500 personnes). Je pense qu'on serait près de l'énoncé anglais en le traduisant par la locution nominative rassemblement de gens en provenance de plusieurs bandes (Leacock 1969: 9-12). Leacock l'emploie pour désigner de grands rassemblements qui se faisaient aux postes de traite français, mais aussi pour circonscrire ceux que décrivirent les missionnaires jésuites allant visiter des nations assemblées en certains lieux durant la saison estivale. Elle précise d'ailleurs que, considérant leur ampleur, ces rassemblements étaient sans doute liés à la présence française, mais qu'ils auraient indubitablement eu des racines profondes au sein de traditions antérieures à la venue des Européens (ibid. : 8-13).

\section{Le récit de Paul Le Jeune et le concept DE « BANDE D'HIVER ॥}

Parmi tous les auteurs des Relations des Jésuites, Paul Le Jeune est sans doute celui qui aura le plus inspiré Leacock, tant est riche et détaillé le récit qu'il a laissé de son séjour avec un groupe d'Innus dont il partagea les expériences quotidiennes durant trois saisons d'affilée. Embarqué le 18 octobre 1633 dans une grosse chaloupe avec son « hôte » - qu'il avait rencontré à Sillery pendant l'été et qui était accompagné de l'un de ses frères, de leurs épouses et de leurs enfants -, Le Jeune put ressentir au tréfonds de luimême les inquiétudes et les angoisses vécues durant les incessantes pérégrinations qu'ils effectuèrent en pourchassant les orignaux et en survivant grâce à la maigre provende que leur fournissait de-ci de-là les castors et les porcs épics. En effet, cet hiver-là fut pénible car il y tomba peu de neige. Or, pour tuer les orignaux avec des arcs ou des harpons, il fallait que des neiges profondes puissent ralentir leur course :

\footnotetext{
Pour la chasse, comme les neiges n'étaient pas profondes à proportion des autres années, ils ne pouvaient pas prendre l'Élan, si bien qu'ils n'apportaient que quelques castors et quelques porcs épics, mais en si petit nombre, que cela servait plutôt pour ne point mourir que pour vivre. Mon hôte me disait dans ces grandes disettes: "Chibiné, aie l'âme dure, résiste à la faim; tu seras parfois deux jours, quelque fois trois ou quatre sans manger, ne te laisse point abattre, prends courage, quand la neige sera venue, nous mangerons. » [...] Quand je pouvais avoir une peau d'anguille pour ma journée, sur la fin de nos vivres, je me tenais pour [avoir] bien déjeuné, bien dîné et bien soupé. (RJ 1972 [1634] : 54)
}

Le récit de Le Jeune est particulièrement intéressant parce que son auteur nous présente de l'intérieur de la maisonnée les hommes et les femmes qui la constituent et dont il fait un portrait clair et vivant en décrivant les relations qui les unissent dans le déploiement de leurs activités quotidiennes. Il contient en outre des données très instructives sur les relations que la famille hôte entretient avec d'autres maisonnées qui cheminent dans les mêmes parages qu'eux depuis le jour où, sur une île située à proximité de la rive, désignée par lui comme «l'île au grand nom $»^{3}$, ils rencontrèrent un Innu appelé Ekhennabamate, que son hôte cherchait à rejoindre. Lhôte apprit de celui-ci que son frère, appelé par Le Jeune le Sorcier, n'était pas loin: il alla le trouver et le ramena avec lui dès le lendemain, $1^{\mathrm{er}}$ novembre. Nous sommes donc en présence de trois frères : il y a l'hôte de Le Jeune, nommé Mestigoït (RJ 1972 [1634] : 58), qui est accompagné de celui qui partit avec eux de Québec, désigné comme l'Apostat (ou le Renégat, car il avait abjuré la foi catholique) et, enfin, il y a le Sorcier, qui est l'aîné des trois. Des Innus, embarqués dans quatre canots, avaient aussi été 
rencontrés un peu plus tôt durant le trajet de navigation et ils avaient dit aux compagnons de Le Jeune que la chasse n'était pas bonne dans les terres sises au nord du fleuve, de sorte que tous ces gens convinrent qu'il serait préférable d'hiverner sur la rive sud (ibid. : 64). Le groupement qui se forme sur l'île (et dans les environs) présente alors la composition suivante :

À notre entrée dans les terres, nous étions trois cabanes de compagnie : il y avait dix-neuf personnes en la nôtre, il y en avait seize en la cabane du Sauvage nommé Ekhennabamate, et dix dans la cabane des nouveaux venus [rencontrés en canot]; je ne compte point les sauvages qui étaient à quelques lieues de nous ${ }^{4}:$ nous faisions en tout quarante cinq personnes [...]. (RJ 1972 [1634] : 66)

Les trois frères sont tous mariés et ils ont des enfants, dont quelques-uns sont de jeunes chasseurs - probablement des fils du Sorcier, qui est l'aîné. À ma connaissance, Le Jeune ne mentionne qu'en une occasion une femme qui serait l'épouse de son hôte et qu'il décrit d'ailleurs comme « une jeune femme étourdie qu'il tenait avec soi [lui] [...] » (ibid. : 58). Quant à l'Apostat, il en avait eu quatre ou cinq, mais comme il était mauvais chasseur, elles l'avaient toutes quitté (ibid. : 80); pour l'heure, il était marié à « une femme d'une autre nation que la sienne » (ibid. : 58). Trois époux donc, et peut-être autant de familles nucléaires qui forment ensemble une même "cabane» de dix-neuf personnes, dont plusieurs enfants et un jeune Iroquois qui avait été adopté pour lui épargner la mort après avoir été capturé.

Entre le 12 novembre, date d'entrée dans les terres, et la mi-décembre, ce « groupe multifamilial», pour reprendre l'expression de Leacock, reste uni, malgré six «stations », terme qui indique sous la plume de Le Jeune des moments d'arrêts suivis de nouveaux départs. Il explique d'ailleurs la dynamique de ces mouvements de la façon suivante :

La faim nous faisait marcher, mais le mal est qu'elle nous suivait partout où nous allions car nous ne trouvions par tout, ou fort peu, ou point de chasse, en cette station, qui fut la sixième, le Renégat vint me dire que les Sauvages étaient fort épouvantés, et mon hôte m'abordant tout pensif, me demanda si je ne savais point quelque remède à leur malheur. II n'y a pas, me disait-il, assez de neige pour tuer l'orignal; des castors et des porcs épics, nous n'en trouvons quasi point, que ferons-nous ? [...]

Le 24 décembre, veille de la naissance de notre Sauveur, nous décampâmes pour la septième fois. [...] Le lendemain [...] fut pour nous un jour de jeûne, on ne me donna rien du tout à manger; la faim, qui fait sortir le loup du bois, m'y fit entrer plus avant, pour chercher des petits bouts d'arbres que je mangeai avec délices [...] Le même jour de Noël, je m'en allai sur le soir visiter nos voisins, Nous n'étions plus que deux cabanes, celle du Sauvage Ekhennabamate avait tiré d'un autre côté depuis cinq ou six jours, à raison qu'il n'y avait pas assez de chasse pour nourrir tout le monde. Je trouvai deux jeunes chasseurs tout tristes, pour n'avoir rien pris ce jour-là, ni le précédent; ils étaient comme tous les autres maigres et défaits, taciturnes et fort pensifs, comme gens qui ne pouvaient mourir qu'à regret : cela me toucha le cœur. (ibid. : 74)

Cette dynamique s'explique donc d'abord et avant tout «à raison qu'il n'y avait pas assez de chasse pour nourrir tout le monde ». Les estimations que font les chasseurs de la disponibilité des ressources sont donc à la base des processus de démembrement du groupe de chasse quand elles paraissent insuffisantes pour toutes les bouches à nourrir. Après le départ d'Ekhennabamate et de sa famille dans la semaine qui précéda Noël, la maisonnée se subdivisera de nouveau le 6 mars quand on découvrira les traces de quatre orignaux et une grande quantité de huttes de castors. Le Jeune et son hôte partirent vers les castors, alors que le Renégat et le Sorcier prirent la direction des orignaux. Ils se donnèrent rendez-vous sur l'île au grand nom et ils s'y retrouvèrent effectivement un peu moins d'un mois plus tard, le 4 avril. Mais, entre ces deux dates, la maisonnée de Le Jeune parcourt encore énormément de terrain à marche forcée, car on ne trouve plus d'orignaux. Néanmoins, on conserve «les plus beaux et les meilleurs » des castors pour les offrir en festins lors des retrouvailles prévues pour le mois d'avril (ibid. : 82).

Le souci de faire de telles réserves manifeste clairement des formes d'entraide qui englobent aussi bien les gens de sa maisonnée que les occupants des maisonnées voisines, si bien que, considérés en extension maximale, les réseaux d'association économique, de coopération et de résidence commune correspondraient à ce que Leacock appelle la bande d'hiver. Le texte de Le Jeune révèle aussi en de nombreux passages que ces réseaux étaient intrinsèquement liés à des pratiques de soutien mutuel qui se muaient souvent dans les formes du secours en galvanisant l'esprit du don. En voici une première illustration :

Le neuvième [jour] de janvier, un Sauvage venant nous visiter, nous dit qu'un homme et une femme du lieu dont il venait étaient morts de faim, et que plusieurs n'en pouvaient plus. Le pauvre homme jeûna le jour de sa venue aussi bien que nous, parce qu'il n'y avait rien à manger; encore fallut-il attendre jusque au lendemain à dix heures de la nuit, que mon hôte rapportât deux castors, qui nous firent grand bien.

Le jour suivant, nos gens tuèrent le second orignal, ce qui causa partout une grande joie; il est vrai qu'elle fut un peu troublée par l'arrivée d'un Sauvage et de deux ou trois femmes, et d'un enfant que la famine allait bientôt égorger, s'ils n'eussent fait rencontre de notre cabane. [...]

Mais admirez s'il vous plait l'amour que ces barbares se portent les uns aux autres : on ne demanda point à ces nouveaux hôtes pourquoi ils venaient sur nos limites, s'ils ne savaient pas bien que nous étions en aussi grand danger qu'eux, qu'ils venaient nous ôter le morceau de la bouche; au contraire on les reçut, non de paroles, mais avec affection, sans courtoisie extérieure, car les Sauvages n'en ont point, mais non sans charité. On leur jeta de grandes pièces de l'orignal nouvellement tué, sans leur dire autre parole, mistisoukau mangez : aussi leur eut-on fait grand tort d'appliquer pour lors leurs bouches à autre usage. Pendant qu'ils mangeaient, on prépara un festin, auquel ils furent traités à grand plat, je vous en réponds: car la portion qu'on leur donna à chacun, sortait beaucoup hors de leurs ouragans [il s'agit de récipients], qui sont très capables [qui peuvent contenir beaucoup].

(RJ 1972 [1634] : 80) 
En voici une deuxième :

Je coucherai ici un exemple capable de confondre plusieurs Chrétiens. Durant les pressions de notre famine, un jeune Sauvage d'un autre quartier nous vint voir ; il était aussi affamé que nous. Le jour qu'il vint fut un jour de jeûne pour lui et pour nous, car il n'y avait de quoi manger; le lendemain, nos chasseurs ayant pris quelques castors, on fit festin, auquel il fut bien traité; on lui dit en outre qu'on avait vu les pistes d'un orignal, et qu'on irait chasser le lendemain; on l'invita à demeurer, et qu'il en aurait sa part: lui répondit qu'il ne pouvait être davantage [demeurer plus longtemps] ; s'étant donc enquis du lieu où était la bête, il s'en retourna. Nos chasseurs ayant trouvé et tué le lendemain cet élan, l'ensevelirent dans la neige, selon leur coutume, pour l'envoyer quérir au jour suivant. Or pendant la nuit mon jeune Sauvage cherche si bien, qu'il trouve la bête morte, et en enlève une bonne partie sans dire mot; le larcin connu par nos gens, ils n'entrent point en des furies, ne donnèrent aucune malédiction au voleur; toute leur colère fut de se gausser de lui, et cependant c'était presque nous ôter la vie, que de nous dérober nos vivres, car nous ne pouvions récupérer [nos forces]. A quelque temps de là, ce voleur vint nous voir ; je voulus lui représenter la laideur de son crime, mon hôte m'imposa silence, et ce pauvre homme rejetant son larcin sur les chiens, non seulement fut excusé, mais encore [fut] reçu pour demeurer avec nous dans une même cabane. Il s'en alla donc quérir sa femme, qu'il apporta sur son dos, car elle a les jambes sans mouvement; et une jeune parente qui demeure avec lui apporta son petit fils, et tous quatre prirent place en notre petit taudis, sans que jamais on leur ait reproché ce larcin, au contraire on leur a témoigné très bon visage, et on les a traités comme ceux de la maison. (ibid. : 29)

On pourrait me reprocher de faire de l'essentialisme en m'imaginant que la culture innue est intrinsèquement faite d'amour et de compassion, mais ce que je veux faire valoir tient plutôt en ce que ce domaine du sentiment résulterait d'une expérience millénaire ayant mené à des constats irréfragables et voulant que le partage soit une garantie de la survie puisque ce que l'on donne gratuitement aujourd'hui pourra nous être rendu quand on sera à notre tour en position de péril. Mais il n'y aurait pas que ce calcul pragmatique, car le fait même de donner cause le plaisir de réjouir ceux que l'on aime et, sur un plan psychique plus profond, il procure au sujet la jouissance d'être en accord avec les idéaux de sa tradition, puisque le système de croyances, en articulant la mythologie et le rituel, préconise le partage en enjoignant à chacun de se solidariser avec les proches contre toutes les forces de la mort.

\section{DES ARRIÈRE-PLANS MYTHIQUES ET RITUELS COMME PARADIGMES DE LA BONNE CONDUITE}

Des mythes de la tradition orale des Algonquins de Kitcisakik témoignent éloquemment de ces préceptes, et leur évocation nous permettra de faire ressortir les paradigmes d'une pensée qui étaye l'éthique du sujet à travers tout le champ des ordres symboliques des peuples considérés ici. Dans un texte paru il y a quelques années (Leroux 2009 : 89), j'attirais l'attention sur le rituel dit du «festin à tout manger », qui était pratiqué par tous ces peuples, en cherchant à montrer qu'il se théâtralisait à travers une symbolique manifestant une sorte de solidarité organique des commensaux mâles qui ne pouvaient sortir de la tente rituelle dans laquelle ils se trouvaient tant qu'ils n'avaient pas entièrement consommé l'animal auquel ils rendaient hommage. C'est un épisode d'un mythe raconté par Salomon Papatie - et que j'ai analysé dans ma thèse de doctorat (Leroux 2003 : 267-295) - qui m'avait mis sur la piste de ce rituel. Ce récit s'intitule "Ayacawe», ce nom étant celui d'un homme jaloux qui a soupçonné son fils d'avoir eu une relation sexuelle avec sa seconde épouse (ce motif est précisé dans d'autres versions). Mû par une sorte de délire, il emmène son fils sur une île et l'y abandonne. Grâce à l'appui d'êtres secourables, le fils traverse au péril de sa vie les obstacles mis en place par son père afin d'empêcher son retour. Une fois revenu à la demeure, il tire une flèche enflammée sur son père dont le corps s'embrase. Il l'incite ensuite à plonger dans un récipient rempli de graisse d'ours, ce à quoi obtempère le père en croyant pouvoir éteindre ainsi le feu qui le consume, mais il y meurt en étant entièrement dévoré par les flammes. Si cet épisode fait référence, par l'intermédiaire du récipient de graisse, au festin à tout manger en l'honneur de l'ours, il le fait en inversant complètement les valeurs sémantiques que véhiculait ce rituel: dans celui-ci, les commensaux rendaient hommage à l'ours en célébrant leur appartenance à un même dispositif d'entraide symbolisé par leur participation au cérémoniel, alors que dans ce mythe le fils pousse les antagonismes initiaux jusqu'à l'anéantissement de son propre père.

Cet épisode est d'ailleurs conforme à tous les autres épisodes du récit en ceci que les antagonismes entre Ayacawe et son fils évoquent l'acquisition par ce dernier de pratiques chamaniques qui lui permettront de triompher de son père. Celles-ci sont donc présentées sous un angle négatif, comme elles le sont dans un récit similaire où c'est un gendre qui s'oppose à son beau-père à travers une série de luttes et de duels chamaniques où le gendre triomphera lui aussi de son aîné. Le beau-père s'appelle Kinongé, nom qui signifie «brochet», et il a fait mourir tous ses gendres précédents du fait d'une jalousie effrénée qui l'empêche de céder sa fille en la donnant en mariage. Lévi-Strauss a commenté dans L'Homme nu (1971 : 307-308) de nombreuses variantes de ces mythes en montrant qu'ils appartiennent au cycle des mythes dit du « dénicheur d'oiseaux » qui ouvrait dans Le Cru et le cuit, premier ouvrage des Mythologiques, la longue étude que Lévi-Strauss a menée sur les continents sud et nord-américains.

La version des Algonquins de Kitcisakik présente un épisode montrant Kinongé qui revient chez lui en plein hiver, nu-pieds dans la neige. Durant la nuit précédente, il avait tenté de brûler les mocassins et les culottes de son gendre, mais celui-ci avait anticipé la manøeuvre et interverti l'ordre des vêtements. Pour se protéger du froid sur le chemin du retour, Kinongé préleva une sorte de lichen qui pend aux branches de certains arbres résineux, que l'on appelle usnées barbues et que mon narrateur comparait à 
des « poils aux arbres ». Il s'en enveloppa les pieds et les utilisa en guise de chaussures. Ces éléments narratifs rappellent un mythe innu dans lequel le personnage de Tshakapesh accrochait à des arbres les cheveux de ses parents morts. Rémi Savard, qui a analysé des variantes innues de ce mythe, a montré que ce tissu végétal était lié à une pratique funéraire décrite par Le Jeune et qui consistait à préserver les cheveux des parents défunts, le tissu végétal constitué par les usnées barbues et le tissu capillaire constitué par les cheveux y apparaissant comme des équivalents sémantiques qui défient le temps en enveloppant les morts et en témoignant comme reliques de leur mémoire (Savard 1985 : 107-108). Cet épisode de la variante algonquine condense toutes ces images et il apparaît alors que Kinongé se déplace dans la neige comme un mort-vivant, complètement isolé de ses proches. En effet, Kinongé a été abandonné par son gendre après qu'il eut tenté de brûler ses vêtements et, quand il arrive enfin près de sa demeure, il implore sa fille d'attiser le feu, car il souffre affreusement du froid. Or, celle-ci, au lieu d'obtempérer - car elle est excédée par les frasques de son père -, éteint le feu en l'étouffant avec de la neige!

Si nous admettons les enseignements de l'analyse lévistraussienne des mythes voulant que, ce qu'une tradition présente sous des modalités négatives, une autre tradition pourra le présenter sous des modalités positives (voir Hénaff 1991 : 249-254), on peut faire le rapprochement entre cet épisode mythique et un rituel des Cris appelé «walking out ceremony» au cours duquel on présente officiellement un enfant à sa communauté en le faisant marcher dans un trajet hautement symbolique, à l'époque où il effectue ses premiers pas (Tanner 1979: 91 sq). Comme dans l'épisode montrant Kinongé qui abandonne la tente où il a voulu mettre le feu aux vêtements de son gendre, l'enfant cri doit d'abord sortir d'une tente où brûle un feu et se diriger vers un arbre situé tout près. Les petits garçons sont munis d'un fusil jouet ou d'un petit arc et de flèches, alors que l'on met une hache miniaturisée dans les mains des petites filles, car ce rite consiste à mimer leurs futures activités d'homme ou de femme. Ainsi, les garçons feignent de tuer un petit animal dont la carcasse a été déposée au pied de l'arbre, alors que les filles feignent de couper du bois qui servirait de combustible. L'enfant doit faire le tour de l'arbre et il revient ensuite à l'intérieur de la tente où il sera reçu par les membres de sa famille. Il devra alors présenter à un parent ou grand-parent du même sexe que lui l'animal trouvé au pied de l'arbre ou le fagot de bois.

Ce rite symbolise l'insertion de l'enfant dans sa société, c'est-à-dire dans un collectif qui lui donne par avance une place et un rôle selon son sexe, mais il le fait en exprimant la solidarité de tous les membres du groupe à travers le rapprochement des plus jeunes et des plus âgés qui s'unissent les uns aux autres contre les forces du froid et de la mort. Or, l'épisode du mythe de Kinongé évoque ce rite mais en inversant complètement la sémantique, puisque Kinongé a voulu abandonner son gendre en jetant ses vêtements au feu et que, au lieu d'être chaleureusement reçu par les membres de sa famille comme l'est l'enfant cri, il revient chez lui grelottant, cacochyme et abandonné de tous ${ }^{5}$ !

\section{Alliance matrimoniale et COde géographique}

Si ces éléments de la pensée mythique algonquine entrent en correspondance avec le rituel des Cris et les préceptes de partage et de solidarité que le Père Le Jeune avait observés chez les Innus, la structure conceptuelle qui se dégage de ce champ sémantique paraît se recouper aussi avec ce que j'appellerais des éléments de codification géographique (cf. Lévi-Strauss 1968: 137). Le mythe de Kinongé est particulièrement révélateur à cet égard en tant qu'il appartient à ce cycle de mythes que Lévi-Strauss regroupait à l'enseigne de la «transformation Putiphar », laquelle se caractérise par un axe de disjonction horizontal opposant des individus appartenant normalement à la catégorie des "alliés », comme le sont un beau-père et son gendre. En analysant ce mythe, je m'étais d'abord interrogé sur l'usage des noms de poissons qui étaient donnés aux personnages, car si le beau-père est, comme on l'a vu, porteur d'un nom équivalent à celui de Brochet, son gendre est par ailleurs nommé Esturgeon. On notera aussi qu'une conteuse du lac Barrière, de qui j'ai entendu une version de ce mythe, disait que Kinongé avait tué six autres gendres avant d'être vaincu par Esturgeon et elle ajoutait qu'ils portaient tous des noms de poissons (Leroux 2003: 171). Pour interpréter le développement de ce champ sémantique, il parut idoine d'étudier le comportement des poissons durant la saison de fraie puisque le mythe pose, par référence à leur comportement locomoteur, des problèmes qui concernent la reproduction humaine sur le plan des relations matrimoniales. Il en est ressorti que le brochet réel (dit Grand Brochet [Esox lucius]) présente un comportement plutôt « sédentaire » selon Scott et Crossman (1978), ce qui l'opposerait à l'esturgeon de lac (Acipenser fulvescens) qui peut parcourir durant cette période des distances de « 80 à 250 milles » (ibid. : 91) [soit de 128 à $400 \mathrm{~km}$ ], mais la distance de 80 milles serait la plus usuelle. Considérant qu'Esturgeon est le seul des sept gendres qui parvient à triompher de Brochet, la pensée mythique lui donne un certain ascendant et il faudrait aussi tenir compte du fait que le personnage de Brochet serait en outre disqualifié comme «modèle » parce que l'espèce qu'il représente se comporte en "cannibale », ce poisson mangeant la progéniture de sa propre espèce (ibid. : 391).

Comme Lévi-Strauss l'a aussi montré dans la troisième partie de L'Origine des manières de table, intitulée « Le voyage en pirogue de la lune et du soleil », la pirogue et les voyages fluviaux servent la pensée mythique à « arbitrer » la bonne distance à franchir pour trouver les partenaires de l'alliance matrimoniale. Dans cette perspective, la pensée mythique algonquine aura pu retenir que la distance la plus souvent parcourue par l'esturgeon pendant la saison du frai serait le 
bon étalon de référence afin de conceptualiser la distance idéale à parcourir pour trouver un conjoint. Peut-être s'agirait-il d'un rayon de 80 milles, ou encore de la moyenne qui se trouve entre les 80 à 250 milles que peut parcourir l'esturgeon, mais, quoi qu'il en soit, on pourrait faire l'hypothèse que pour la pensée mythique les réseaux d'alliances matrimoniales se recoupaient avec les routes fluviales que configuraient les principales rivières reliant les différentes bandes locales. On se rappellera, comme on l'a vu plus haut, que les noms de bandes locales chez les Innus correspondent très souvent aux bassins hydrographiques qu'elles occupent durant une bonne partie de l'année et on pourrait penser que l'alliance matrimoniale reposait sur un circuit préexistant de communication, soit le réseau hydrographique de chaque région qui « orientait» par avance l'intérêt des familles voulant marier leurs enfants vers les groupes avec lesquels on privilégiait des alliances. Grâce à l'usage des canots, les distances pouvaient être plus rapidement franchies de sorte que les contacts entre ces unités étaient plus nombreux et plus intensifs durant la saison estivale. Ce point sera plus amplement développé dans le deuxième article, mais on pourrait avancer immédiatement l'idée que le cercle des unités contractantes de l'alliance matrimoniale pouvait s'établir à partir d'un centre constitué par la famille d'Égo.

On pourrait rapporter les plus petites unités à celles que Mailhot appelle «bandes locales », lesquelles correspondraient aussi, en partie, à ce que Leacok appelle «bande d'hiver », si l'on admet, après lecture du texte de Le Jeune, que celle-ci se recoupe avec un réseau d'interactions sociales qui maintiennent à plus ou moins grande distance des maisonnées cheminant en parallèle et qui se soutiennent les unes les autres dans les formes du don et de l'entraide. Lunité de niveau supérieur qui les intègre dans un réseau d'alliances pourrait être rapportée à celle que Leacock appelait « le groupe nommé ou la bande », mais il ne faudrait pas concevoir cette unité comme si elle existait dans un circuit fermé et je m'efforcerai dans un prochain article de voir comment on peut la différencier de la bande locale (ou «bande d'hiver »). Dans ces perspectives, on peut alors poser la question suivante: quels sont les rapports entre les réseaux de solidarité et l'espace territorial?

\section{« QUARTIERS " ET « DISTRICTS "}

Les études ethnologiques sur le calendrier des activités saisonnières des Innus montrent qu'en dépit d'une intensification des activités de piégeage qui se serait amorcée à des époques différentes selon les régions, des rassemblements réunissant des dizaines de familles s'effectuaient encore dans les premières décennies du $\mathrm{Xx}^{\mathrm{e}}$ siècle. La lecture de ces documents donne donc à penser que le groupement défini par Leacock comme winter band a perduré, mais il faudrait maintenant poser que cette unité sociale se manifestait en plusieurs autres moments de l'année. Ainsi, dans un texte rédigé conjointement avec Loren C. Eiseley, Speck citait un de ses propres rapports, rédigé en 1931, concernant la «bande de Michikamau », où il apparaît que l'alternance des activités de piégeage et de chasse au caribou rythmait alors les mouvements de fractionnements et de recomposition du groupe :

\begin{abstract}
Les Indiens de Michikamau vivent et chassent continuellement [en formant] une communauté de familles regroupées. Ce n'est que lorsqu'ils sont sous la pression de la famine qu'ils se séparent et vivent de petit gibier; sous la juridiction du chef, le groupe comprend treize chefs de familles qui sont pratiquement toutes reliées par consanguinité et mariage.
\end{abstract}

Jusqu'à récemment, cette bande allait à North-West River pour y faire la traite. Maintenant, ses membres font ensemble, chaque année, la longue et dangereuse descente depuis leur lac [Michikamau] pour atteindre le poste de Sept-îles en passant par les lacs Menihek, Ashwanipi et la rivière Moisie. [...]

(Speck et Eiseley 1942 : 234)

Le chef Sylvestre Mackenzie a exposé les étapes principales de ce voyage effectué en 1924 en le décrivant en sens inverse, c'est-à-dire par la remontée dans les terres :

\begin{abstract}
Ponctuellement, la bande quitte Sept-îles le $1^{\mathrm{er}}$ août en remontant la rivière Moisie [et] en atteignant le lac Menihek vers le 5 octobre. Ils y campent, pêchent et chassent durant quelques jours. Ils poursuivent ensuite la route, en chassant et en pêchant, atteignant le lac Michikamau à la fin du mois d'octobre; de là, ils planifient de se séparer en groupes familiaux pour une saison de piégeage en vue d'accumuler des fourrures. Il est essentiel, avant cette dispersion temporaire, qu'ils décident du lieu où ils se rencontreront pour le rendez-vous d'hiver. II a parfois lieu en novembre. À partir de ce moment et jusqu'à la fin de janvier, ils circulent en formant une bande qui se nourrit du caribou en suivant ses déplacements. C'est la période de chasse mi-hivernale. À la fin janvier survient la période la plus critique si le caribou venait à manquer et le groupe se fractionne de nouveau, les familles allant reprendre leurs activités de piégeage dans les aires qu'elles fréquentent habituellement [in their habitually frequented tracts]. Vers la fin du mois de mars ou au commencement du mois d'avril, le groupe en son entier [the entire 'gang' (a trader's term)] se présente de nouveau au rendez-vous habituel du lac Menihek. Alors se crée un campement étendu de familles occupant des tentes [et l'on se prépare alors à redescendre vers Sept-îles où l'on arrive vers le 25 juin]. (ibid. : 234-235)
\end{abstract}

Les auteurs mentionnent ensuite que les chefs de famille occupent des zones où ils ont l'habitude de faire du piégeage et ils précisent que...

[le] chef Mackenzie déclarait que leurs emplacements étaient décidés sous son approbation et en fonction de l'assentiment général des autres [chefs de famille], lesquels avaient en tête le bienêtre de la bande entière [the whole horde] [en prévision] des périodes les plus dures de l'hiver (ibid. : 236).

Ces mouvements de dilatation et de concentration du groupe ont aussi été mentionnés par David Denton qui avait fait une étude dans «la région de Caniapiscau», qui se recoupe en partie avec celle où Speck avait mené son enquête (Denton 1979 : 110). 
Je souligne que Leacock ne limitait pas la composition de la bande d'hiver à cette seule saison, puisqu'elle la présentait en écrivant que plusieurs maisonnées quittaient les rives du fleuve à l'automne en cheminant vers l'intérieur des terres et qu'elles se voyaient ensuite forcées de se séparer à la fin du mois de décembre pour chasser sur de vastes étendues de terres (Leacock 1969 : 9). Son modèle était élaboré à partir du récit de Le Jeune, mais aussi de ce qu'elle tirait des lectures de Druillettes, Albanel et Crépieul, autres jésuites qui avaient hiverné avec des Innus. Il faut dire que dans le cas de Le Jeune et de Druillettes, les maisonnées multifamiliales n'étaient pas sur leurs terres de chasse habituelles, et cette particularité nous empêche de bien saisir l'ancrage des maisonnées et de la bande locale sur les territoires qu'elles occupaient. Le récit d'hivernement du Père Crépieul, lui, se déroule sur les aires de chasse habituelles, entre le Saguenay et le lac à la Croix (situé à $70 \mathrm{~km}$ de la rive occidentale de l'actuel réservoir Manicouagan) (ibid. [1672] : 27-31). On peut aussi ajouter à ce récit celui du Père Buteux effectué chez les Attikameks, à la fin duquel trois bandes de cette nation se rencontrent pour assister à une grande assemblée (ibid. [1651] : 15-26). Le voyage du Père Nouvel - qui le mena, en juin 1664, au lac Manicouagan chez les Papinachois « venus faire leur trafic avec leurs compatriotes qui habitent le long du grand fleuve St-Laurent » (ibid. [1664] : 15) -, indique des rapports de communication entre gens de l'amont et gens de l'aval, mais on ne saurait dire si de telles rencontres se faisaient depuis l'établissement des postes français puisque leur présence aurait pu stimuler le troc de biens achetés dans ces postes, mais un schème de déplacements entre les gens de l'intérieur des terres et ceux de l'aval est attesté depuis au moins les années 1840 :

L'intérieur du pays est habité par un nombre inconnu de petits groupes dont l'économie est axée sur la chasse au caribou et aux animaux à fourrures, ce sont principalement eux qui fournissent la compagnie de la baie d'Hudson en pelleteries. On les appelle à l'époque les Montagnais de l'intérieur ou Montagnais des terres ou encore "Naskapis», dans le sens de "barbares» ou "noncivilisés ». [...] Les renseignements sont peu précis en ce qui concerne le nombre de groupes de Montagnais des terres qui occupent I'arrière-pays de la Côte-Nord. Nous savons que les rivières aux Outardes, Manicouagan, Sainte-Marguerite et Moisie constituent de grandes voies d'accès vers l'intérieur et que tout leur cours est occupé par des Montagnais chasseurs de caribous et d'animaux à fourrures. (Mailhot 1996 : 327)

Quoi qu'il en soit de l'ancienneté historique de ces déplacements quant à leur étendue et leur fréquence, les Jésuites évoquent très souvent la relation intrinsèque entre des groupes et des secteurs qu'ils habitent. Parfois même, c'est l'association entre des individus et de tels secteurs qui est faite, comme dans un récit du Père Buteux relatant son voyage en Mauricie avec un "capitaine» (désigné aussi comme « mon hôte ») et une quarantaine de personnes (comprenant des enfants et des adultes) [RJ 1972 [1651] : 16]. Ainsi, après avoir progressé péniblement dans les neiges fondantes du printemps et sur des glaces fragiles, il mentionne qu'après « avoir traversé quatre lacs, nous arrivâmes à celui où mon hôte fait sa demeure la plus ordinaire » (ibid. : 19, c'est moi qui souligne), soit, d'après ce que l'on peut vraisemblablement en déduire, un secteur qu'il a l'habitude de fréquenter. Cependant, de tels secteurs sont plus souvent associés à des groupes qu'à des individus. Par exemple, lors du voyage de Henry Nouvel dans la région du lac Manicouagan en 1664, celui-ci mentionne qu'après avoir quitté l'île aux Basques, ses compagnons et lui traversèrent sur la rive nord du fleuve et arrivèrent à la rivière Esseigou, (probablement la rivière aux Escoumins), étape à partir de laquelle ils se dirigèrent vers l'est pour ensuite monter vers le lac Manicouagan. Chemin faisant, ils tuèrent cinq orignaux, et les Papinachois eux-mêmes associèrent cette contrée à la leur :

Ce fut alors que les Papinachois, glorieux de cette chasse, me dirent: quelques Montagnais t'ont dit que notre pays est un méchant pays; que tu y mourrais de faim si tu venais avec nous; tu vois maintenant qu'ils n'ont pas dit vrai: Kataouatichouasti Oupapnnachiouek asti, asti, c'est une bonne terre, disaient-ils, que la terre des Papinachois. (RJ 1972 [1664] : 13)

Peu après ce passage, Nouvel emploie l'expression « quartiers » pour désigner des secteurs habités par des populations relativement bien identifiées, au moment où ils rencontrent le père Druillettes, accompagné d'autres Français :

Ce père et moi ayant conféré sur ce que nous avions à faire touchant nos missions, nous conclûmes quej'accompagnerais les Papinachois dans leur voyage des terres, et que le père monterait dans le Saguenay, pour visiter les sauvages de ces quartiers-là, après quoi nous nous séparâmes. (ibid. : 14, je souligne)

Parfois, les Jésuites donnent le nom de « bandes » à ces petits groupes, comme on le verra ici, et ils remplacent aussi le mot « quartiers » par celui de « district », les deux termes y apparaissant comme des synonymes:

[...] il tomba tant de neige, qu'ils en eurent suffisamment pour tuer leurs grandes bêtes, mais comme ils en trouvaient peu, ils furent contraints de se séparer en deux bandes, Georges Etouet Capitaine de Tadoussac, donna le quartier le plus abondant en chasse à Noel Negabamat, par une charité vraiment chrétienne, et par une coutume qui n'a rien de barbare au milieu de la barbarie, c'est que les capitaines d'un pays, donnent toujours l'avantage aux capitaines des autres nations ${ }^{6}$, qui viennent chasser en leur district. [...]

Mais pour ne pas m'écarter de mon chemin, comme les chasseurs de ce capitaine [Georges Etouet] trouvaient de quoi vivre passablement, quatre cabanes d'un autre quartier vinrent se jeter entre leurs bras, criant à la faim, parce qu'il n'y avait ni élans ni castors, disaientils, dans leur district. [..] II n'est pas croyable combien les Sauvages sont charitables en ces rencontres, on ne tança point ces bonnes gens, de ce qu'ils couraient sur les marches d'autrui, on leur fait part de tout ce qu'il y a dans ces cabanes [...]

(RJ 1972 [1648] : 30, je souligne)

[...] ils troussent bagage, ils lèvent le camp, et leur premier pas est vers la chapelle, où ils vont prendre la bénédiction de Notre Seigneur, et ensuite chacun tire vers son quartier d'hiver; n'allant 
néanmoins qu'aux endroits dont ils ont convenu, avant que de se séparer les uns des autres. Pour les Pères, ils se retirent à Québec. Quelques-uns se joignent parfois aux plus grosses bandes, pour les instruire dans ces profondes forêts, où on ne rencontre que des arbres, des glaces, et des neiges [...]. (RJ 1972 [1652] : 12, je souligne)

Récapitulons: le mot «quartier» a d'abord, dans le texte de H. Nouvel, une extension assez générale (« dans ces quartiers-là ») en désignant une région habitée par des Innus du Saguenay, mais le terme prend une extension plus restreinte quand il est dit que Georges Etouet en donna un à Noël Negabamat, sans doute en considérant que celui-ci était à la tête d'une maisonnée multifamiliale, car l'auteur parle ensuite de "quatre cabanes d'un autre quartier», ce terme désignant alors l'aire exploitée par des familles formant une petite bande d'hiver. Le mot «quartier» était aussi employé par des auteurs anglophones sous la forme de "quarter», comme on peut le voir dans un texte de Rogers (1969), qui mentionne que l'auteur qu'il cite (Oldmixon 1741) y discute de patterns d'occupation et du rôle des chefs chez les «Indiens de la baie James »:

II n'a aucune autorité mais ce qu'ils pensent s'ajuste à l'occasion pour lui en donner. II est leur porte-parole auprès des Anglais aussi bien que dans leurs délibérations les plus sérieuses, quand ils se rencontrent au printemps et à l'automne pour planifier leur répartition dans leurs quartiers de chasse et de pêche. Les familles ajustent ainsi leurs frontières, parfois en les quittant quand elles ne trouvent pas de succès à la chasse et qu'elles se joignent alors à d'autres familles qui en ont trouvé. (Oldmixon 1741, cité par Rogers 1969 : 40-41)

Ce texte présente ceci de particulièrement intéressant qu'Oldmixon associe clairement des aires à des familles précises (i.e. des maisonnées multifamiliales), mais en même temps il révèle que la totalité du territoire de la bande fait l'objet de discussions collectives puisque les familles choisissent de s'établir en tel ou tel secteur en fonction de la disponibilité des ressources. Il apparaît donc qu'une gestion collective du territoire n'est nullement incompatible avec l'existence d'une aire habituellement fréquentée par une ou plusieurs maisonnées. On se rappelle qu'un tel dispositif de gestion ressortait aussi du texte de Speck qui mentionnait que le calendrier annuel de la bande de Michikamau comprenait des regroupements ponctuels de plusieurs familles qui s'effectuaient en alternance avec des moments de dispersion sur des territoires qu'elles avaient l'habitude de fréquenter. En fait, les intérêts spécifiques des maisonnées en regard de la disponibilité des ressources sur les aires qu'elles occupaient n'étaient pas évalués par le petit bout de la lorgnette, comme si ceux-là seuls comptaient, car le souci de protéger et d'entretenir la ressource s'envisageait à long terme et en fonction d'une focale qui impliquait les vues et la concertation de plusieurs acteurs de la bande. Dans un travail mentionné plus haut, j’avais défini comme «maîtrise prioritaire » la compétence (terme qu'il faut entendre en son sens juridique) d'un leader de maisonnée et de ses proches à occuper une aire spécifique, cette prérogative s'accompagnant de facto d'une proximité spatiale facilitant l'accès à la ressource (Leroux 2010 : 117). Je l'avais aussi insérée dans un modèle conceptuel voulant que l'attribution d'une aire de chasse au leader ait été concomitante de pratiques de protection dans la mesure où son occupation et son exploitation impliquaient que lui et sa famille agissent de façon responsable quant à sa préservation (ibid. : 126-127). Or, cette maîtrise peut aussi se partager grâce à des modes de cohabitation et de collaboration, ainsi que Speck l'avait montré à propos des chasseurs de Mistassini qui avaient mis en place ce qu'il appelait un «système d'échanges des privilèges de chasse» (Speck 1917: 91). Adrian Tanner avait d'ailleurs repris ce concept en montrant que certaines mesures de protection de la ressource consistaient à laisser en jachère pendant un an ou deux des portions de l'aire occupée par une maisonnée afin de permettre aux castors et autres animaux à fourrure de s'y maintenir sans compromettre la reproduction de l'espèce. Il exposait que les familles y procédaient en libérant des portions de leur propre aire d'occupation mais que ces mesures se couplaient à un partage des prérogatives à travers des pratiques de corésidence impliquant l'union de deux ou trois leaders et de leurs familles au sein d'une même maisonnée (Tanner 1971: 78; 1978: 195-197). Ces pratiques sont d'ailleurs très bien illustrées dans le film Chasseurs cris de Mistassini (Richardson et Ianzelo 1974), où l'on voit effectivement trois leaders et leurs familles cohabiter dans la même maison durant tout un hiver? .

Comme on l'a vu en lisant le récit d'hivernement du Père Le Jeune, les chefs de famille devaient mettre en balance la quantité de biomasse animale disponible à tel ou tel moment en corrélant cette donnée au volume des effectifs humains présents dans les maisonnées. Dans cette perspective, il est logique d'induire qu'il fallait aussi tenir compte des maladies qui auraient pu affaiblir certaines familles, des accidents qui auraient pu handicaper des chasseurs, des morts qui auraient pu survenir à la suite de famines, bref de prendre en considération la composition effective des maisonnées en envisageant de secourir les plus démunis par leur intégration à d'autres groupes, ou, à l'inverse, en y intégrant des effectifs masculins et féminins qui pouvaient suppléer aux manques éprouvés par certaines familles. Si l'on considère qu'une bande locale comprenait de cinquante à soixante-quinze personnes étroitement apparentées, il est indéniable que le collectif qui prenait de telles décisions au printemps et à l'automne, ainsi que le disait Oldmixon, devait comprendre une bonne partie de cette population et rien n'empêche de penser que de telles décisions pouvaient se prendre à n'importe quel moment de l'année, si tant est que ces familles pouvaient communiquer assez facilement entre elles du fait de leur proximité spatiale.

\section{RÉTROSPECTIVE ET ANTICIPATIONS}

Pour conclure, la bande d'hiver se manifeste comme un réseau d'entraide entre maisonnées qui se lient les unes aux 
autres sur la base d'intérêts permanents. En cela il faudrait la définir autrement que par la seule référence à la saison hivernale. Elle présente en outre de nombreux points communs avec l'unité que José Mailhot appelle bande locale, et il serait pertinent d'envisager d'autres similarités - ou de relever des différences - en envisageant les relations qu'elle entretient avec des unités de même niveau, ou de niveaux supérieurs qui les incluraient. Les références à des mythes algonquins donnent à penser que les schèmes d'alliances matrimoniales pouvaient s'organiser autour d'unités similaires dans l'Outaouais supérieur; or, en orientant plus directement l'analyse vers cette région, il s'agira de voir dans le prochain article comment les groupes sont nommés ou se nomment eux-mêmes en assumant des critères d'identité et de reconnaissance mutuelle, ce qui impliquera de considérer des processus de symbolisation se réalisant à travers diverses formes de participation religieuse et politique.

\section{Notes}

1. Un troisième article s'intitulera «Contribution à l'étude de l'effacement de la parenté croisée dans les missions catholiques » et j'en prévois un quatrième qui présentera une synthèse des précédents.

2. En estimant qu'une famille nucléaire comptait environ cinq personnes chez les peuples algonquiens des régions considérées, on pourrait avancer que les bandes locales de ces régions comptaient en moyenne entre 50 et 65 personnes. Par exemple, Speck et Eiseley dénombraient « 10 familles » pour la bande de Moisie («Ashuanipi ») [1942 : 225], 13 familles pour la bande de Caniapiscau (ibid. : 229-230) et 13 « chefs de famille» pour la bande de Michikamau (ibid. : 234).

3. Cette île était appelée par les Innus Ca pacoucachtechckhi chachagou achiganikhi, Ca pakhitaouananiouikhi (RJ 1972 [1634] : 62). Le Jeune précise qu'après y avoir laissé leurs embarcations, la rive sud du fleuve fut atteinte en traversant une "prairie » à marée basse. Moreau estime à environ $80 \mathrm{~km}$ la distance parcourue par voie fluviale depuis Québec (Moreau 1980: 43) jusqu'à ce point d'arrivée. En se donnant beaucoup de latitude, on pourrait estimer que cette île se trouve quelque part entre l'actuel village de Trois-Saumons et celui de Saint-Roch-desAulnaies. La description des îles précédentes le donne à penser (en particulier l'île aux Oies), mais cela resterait à vérifier en visitant les îles de la région, car Le Jeune la décrit en disant qu'elle « n'a pas une demi lieue de tour [soit environ $2 \mathrm{~km}$ ] [et qu'elle] n'est quasi qu'un grand rocher affreux » (RJ 1972 [1634] : 62).

4. Les «sauvages qui étaient à quelques lieues de nous» avaient été visités par les gens de la famille hôte (ibid. : 61) et on les retrouvera en quelques occasions durant les pérégrinations hivernales.

5. On pourrait énoncer les choses différemment en mentionnant des rapports de corrélations et d'oppositions entre mythes et rites de peuples distincts, si on considère que «les éléments changent de signe quand leur ordre est inversé » (par exemple, le feu disjoncteur dans le mythe algonquin s'inverse en feu conjoncteur dans le rite cri ; l'arbre symbolisant la mort dans le mythe algonquin symbolise la continuité de la vie dans le rite cri, etc.) [Lévi-Strauss 1958 : 262], à quoi l'on pourrait ajouter que, si la marche de l'enfant apparaît dans le registre d'une progression sur le plan des relations intersubjectives, celle de Brochet apparaît clairement dans celui de la rétrogression, « la relation entre les deux systèmes [offrant] un caractère contrapunctique [...]» (ibid. : 263).

6. Noël Negabamat était Algonquin.

7. Dans la présentation qui est faite des familles au début du film, un commentaire parlé informe le spectateur que le chasseur Ronnie Jolly dispose d'un territoire situé à peu près à une trentaine de milles de celui de Sam Blacksmith qui l'a accueilli, et on précise qu'il n'y a pas chassé depuis deux ans « pour y favoriser le repeuplement animal » (Richardson et Ianzelo 1974).

\section{Ouvrages cités}

BOUSQUET, Marie-Pierre, 2008 : «A Question of Emotions and a Matter of Respect: Interpreting Conversion to Catholicism Among Quebec Algonquins », in K.S. Hele et R. Darnell (dir.), Papers of the Thirty-Ninth Algonquian Conference : 52-71. University of Western Ontario, London, Ontario.

_, 2012 : «Aiamie, agir au mieux? Éthique, rituels catholiques et corps social chez les Anicinabek depuis les années $1950 »$. Théologiques 20(1-2) : 385-417.

_, 2015 : «Chamanisme et catégories conceptuelles : points de vue anicinabek (algonquins) ». Anthropologica 57(2) : 315-326.

DAMAS, David, 1969: Contributions to Anthropology: Band Societies. Proceedings of the Conference on Band Organization. Bulletin 228, Anthropological Series 84, National Museum of Canada, Ottawa.

DENTON, David, 1979: "Lexploitation historique du caribou et les schèmes d'établissement dans la région de Caniapiscau ». Recherches amérindiennes au Québec IX(1-2) : 105-115.

GÉLINAS, Claude, 1998 : « Jean-Baptiste Boucher, le négatif du chef atikamekw par excellence au XIx ${ }^{e}$ siècle », in N. Clermont (dir.), Anthropologie et histoire. Actes du quatrième colloque du département d'anthropologie : 27-37. Université de Montréal, Montréal.

—, 2000: La gestion de l'étranger. Les Atikamekw et la présence eurocanadienne en Haute-Mauricie 1760-1870. Septentrion, Sillery.

—, 2003a : Entre l'assommoir et le godendart. Les Atikamekw et la conquête du Moyen-Nord québécois. 1870-1940. Septentrion, Sillery.

_, 2003b: «Les missions catholiques chez les Atikamekw (18371940): manifestations de foi et d'esprit pratique». Études d'histoire religieuse 69 : 83-99.

HÉNAFF, Marcel, 1991 : Claude Lévi-Strauss et l'anthropologie structurale. Belfond, Paris.

INKSETTER, Leila, 2015 : «Le chef gagne tous les jours de nouveaux sujets ». Pouvoir, leadership et organisation sociale chez les Algonquins des lacs Abitibi et Témiscamingue au $19^{e}$ siècle. Thèse de doctorat (Ph. D), département d'anthropologie, Université de Montréal, Montréal.

LEACOCK, Eleanor, 1969: «The Montagnais-Naskapi Band », in David Damas (dir.) Contributions to Anthropology: Band Societies. Proceedings of the Conference on Band Organization: 1-17. Bulletin 228, Anthropological Series 84, National Museum of Canada, Ottawa.

LEGENDRE, Pierre, 2004 [1985] : Linestimable objet de la transmission. Étude sur le principe généalogique en Occident. Fayard, Paris.

LEROUX, Jacques, 2003 : Cosmologie, mythologie et récit historique dans la tradition orale des Algonquins de Kitcisakik. Thèse de doctorat (Ph. D), département d'anthropologie, Université de Montréal. Montréal. 
_, 2009: «Éthique et symbolique de la responsabilité territoriale chez les peuples algonquiens du Québec ». Recherches amérindiennes au Québec XXXIX(1-2) : 85-97.

— 2010 : «Le contrôle territorial ou posséder ce que l'on protège chez trois peuples algonquiens du Québec », in Pierre Noreau (dir.), Gouvernance autochtone: reconfiguration d'un avenir collectif. Nouvelles perspectives et processus émergents: 99-131. Les Éditions Thémis, Montréal.

LEROUX, Jacques, Roland CHAMBERLAND, Edmond BRAZEAU et Claire DUBÉ, 2004 : Au Pays des peaux de chagrin. Occupation et exploitation territoriales à Kitcisakik (Grand-Lac-Victoria) au $X X^{e}$ siècle. Les Presses de l'Université Laval et le Musée canadien des civilisations, Québec/Gatineau.

LÉVI-STRAUSS, Claude, 1958: «Structure et dialectique». Anthropologie structurale : 257-266. Plon, Paris.

—, 1968 : L'Origine des manières de table. Plon, Paris.

_, 1971 : L'Homme nu. Plon, Paris.

MAILHOT, José, 1993: Au pays des Innus: les gens de Sheshatshit. Coll. Signes des Amériques 9, Recherches amérindiennes au Québec, Montréal.

—, 1996: «La marginalisation des Montagnais», in Pierre Frenette (dir.), Histoire de la Côte-Nord: 321-357. Institut québécois de recherche sur la culture/Presses de l'Université Laval, Québec.

MOREAU, Jean-François, 1980 : "Réflexions sur les chasseurscueilleurs. Les Montagnais décrits par Le Jeune en 1634 ». Recherches amérindiennes au Québec X(1-2) : 40-49.

OLDMIXON John, 1741 : The British Empire in America, containing the history of the discovering, settlement, progress and state of the British Colonies on the continent and the islands of America. 2 vol. J. Brotherton, J. Clarke [et al.], Londres.

RJ, 1972 [1611-1672]: Relations des Jésuites. Éditions du Jour, 6 tomes, Montréal.

RICHARDSON, Boyce, et Tony IANZELO, 1974: Chasseurs cris de Mistassini. Film cinématographique, son, coul., 74 min. Office national du film du Canada, Montréal. <https://www.onf.ca/ film/chasseurs_cris_de_mistassini/> (consulté le 23 janvier 2017).

ROGERS, Edward S., 1962: The Round Lake Ojibwa. Art and Archaeology Division Occasional Paper 5, Royal Ontario Museum, Toronto.

—, 1963: The Hunting Group-Hunting Territory Complex Among the Mistassini Indians. Bulletin 195, Anthropological Series 63, National Museum of Canada, Ottawa.

—, 1969: «Band Organization among the Indians of Eastern Subarctic Canada», in David Damas (dir.) Contributions to Anthropology: Band Societies. Proceedings of the Conference on Band Organization: 21-50. Bulletin 228, Anthropological Series 84, National Museum of Canada, Ottawa.

ROGERS, Edwin S., et Eleanor LEACOCK, 1981 : « MontagnaisNaskapi », in June Helm (dir.), Handbook of North American Indians, vol. 6, Subarctic: 169-195. Smithsonian Institution, Washington, D.C.

SAVARD, Rémi, 1985 : La Voix des autres. L'Hexagone, Montréal.

SCOTT, W. Beverley, et Edwin J. CROSSMAN, 1978 : Poissons d'eau douce du Canada. Bulletin 184, Environnement Canada, Service des pêches et des sciences de la mer, Office de recherches sur les pêcheries du Canada, Ottawa.

SPECK, Frank G., 1917: "The social structure of Northern Algonkian ». Papers and Proceedings of the American Sociological Society, vol. $12: 82-100$.

SPECK, Frank G., et Loren C. EISELEY, 1942: «MontagnaisNaskapi Bands and Family Districts of the Central and Southeastern Labrador Peninsula ». Proceedings of the American Philosophical Society 85 : 215-242

TANNER, Adrian, 1971 : «Existe-t-il des territoires de chasse? » Recherches amérindiennes au Québec I (4-5) : 69-83.

—, 1979: Bringing Home Animals: Religious Ideology and Mode of Production of the Mistassini Cree Hunters. Institute of Social and Economic Research, Memorial University of Newfoundland, St. John's. 